\title{
Performance Evaluation of Underwater Wireless Optical Communications Links in the Presence of Different Air Bubble Populations
}

\author{
Hassan Makine Oubei, ${ }^{1}$ Member, IEEE, Rami T. ElAfandy, ${ }^{1}$ Ki-Hong Park, ${ }^{2}$ Member, IEEE, Tien Khee Ng, ${ }^{1}$ \\ Member, IEEE, Mohamed-Slim Alouini, ${ }^{2}$ Fellow, IEEE, and Boon S. Ooi ${ }^{1}$ \\ ${ }^{I}$ Photonics Laboratory, Computer, Electrical and Mathematical Sciences and Engineering Division, King Abdullah University of \\ Science and Technology (KAUST), Thuwal 23955-6900, Kingdom of Saudi Arabia \\ ${ }^{2}$ Communication Theory Laboratory, Computer, Electrical and Mathematical Sciences and Engineering Division, King Abdullah University of \\ Science and Technology (KAUST), Thuwal 23955-6900, Kingdom of Saudi Arabia
}

\begin{abstract}
We experimentally evaluate the performance of underwater wireless optical communication (UWOC) links in the presence of different air bubbles. Air bubbles of different sizes and densities are generated by using an air pipe in conjunction with a submersible water pump of variable flow rate that help break up large bubbles into smaller bubbles. Received signal intensity measurements show that bubbles significantly degrade the performance of UWOC links. Large bubbles completely obstruct the optical beam and cause a deep fade. However, as the bubble size decreases, the level of deep fade also decreases because the optical beam is less susceptible to complete obstruction and more light reaches the detector. We also show that beam expansion could help mitigate the performance degradation due to the deep fade caused by air bubbles scatters in the channel.
\end{abstract}

Index Terms: Oceanic propagation, underwater wireless optical communication (UWOC), optical wireless communication (OWC), visible light communication (VLC), diode lasers, oceanic turbulence

\section{Introduction}

UWOC has gained a lot of interest during the last decade and has been proposed as an alternative or complementary solution to the existing acoustic and radio frequency (RF) underwater wireless communication technologies. Using efficient and low-cost light sources such as light-emitting diodes (LEDs) [1] and laser diodes (LD) [2] in the low absorption 400-550 nm window of the electromagnetic spectrum in seawater, UWOC can provide orders of magnitude more bandwidth and achieve much higher data rates for short and moderate ranges $(<100 \mathrm{~m})[3,4]$. In UWOC links, the primary factors that degrade system performance are absorption, scattering, and turbulence. In absorption, the photon energy is lost due to the interaction of light with water molecules and other particulates. Scattering is the process whereby the photons are scattered away from the initial path after interacting with particles and bubbles in the water. Underwater optical turbulence (UOT) is defined as random index-of-refraction variations that affect the beam as it propagates through a water column. The index inhomogeneity can be due to localized temperature, salinity or pressure changes in the water [5].

In recent years, there has been a significant progress of research on UWOC both theoretically and experimentally. However, many relevant studies have mostly focused on the effects of absorption and scattering by particles on the optical channel [6-9], system level design and demonstrations [10-13], underwater turbulence [14-16], and there is little study on the effect of air bubbles in UWOC channels [17]. Air bubbles in the oceans are mainly produced by breaking surface waves and rain [18]. They come in different sizes and assume spherical shape in water as reported by several authors [19-21]. Air bubbles contribute to many oceanographic processes such as propagation and scattering of acoustic waves [22], organic-particle formation, and bacteria transport [23]. When the optical signal propagates through a bubbly underwater channel, the signal's amplitude is distorted due to reflection and refraction at the waterbubble's interface that causes the light to stray into various directions. Thus, in the presence of air bubbles, the scattering properties of the water body are significantly affected. Therefore, it is important to have a detailed understanding on how different sizes of air bubbles affect the performance of UWOC systems. In this paper, we experimentally study the effect of different air bubble populations on UWOC system performance. 


\section{Experimental Setup}

Figure 1 shows the experimental setup used to evaluate the performance of UWOC links in the presence of different air bubble populations. The main experimental platform consisted of a glass water tank of dimensions $50 \mathrm{~cm} \times 34$ $\mathrm{cm} \times 34 \mathrm{~cm}$. The water tank is three-fourths filled with either fresh tap water or Harbor II water. The bubble generation was ensured by a $1 / 4$ " copper pipe with $1 \mathrm{~mm}$ hole directly connected to the Laboratory dry air system as shown in Fig. 1(a). The air flow rate was maintained at around 1.04 standard liters per minute (2.2 SCFH) throughout the experiment. In addition, we used a submersible fountain pump (Haibao HB103) in front of the air pipe to break up and produce bubbles in four different size ranges by changing the pump flow rate. Fig. 1(b) illustrates the schematic of the experimental setup.

(a)

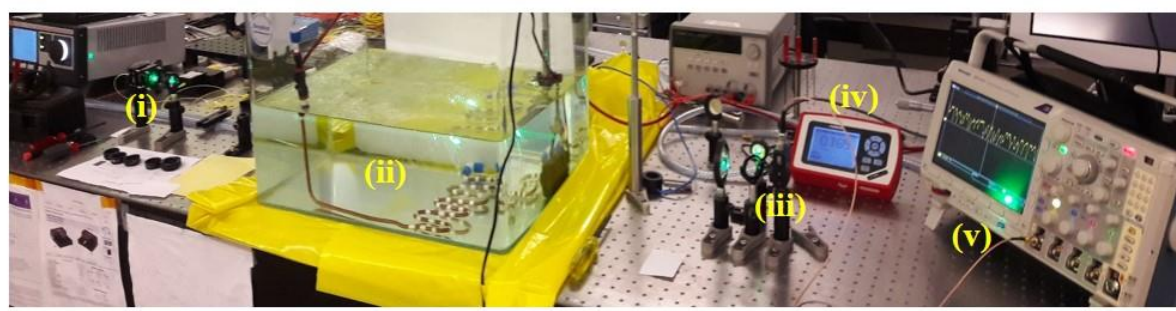

(b)

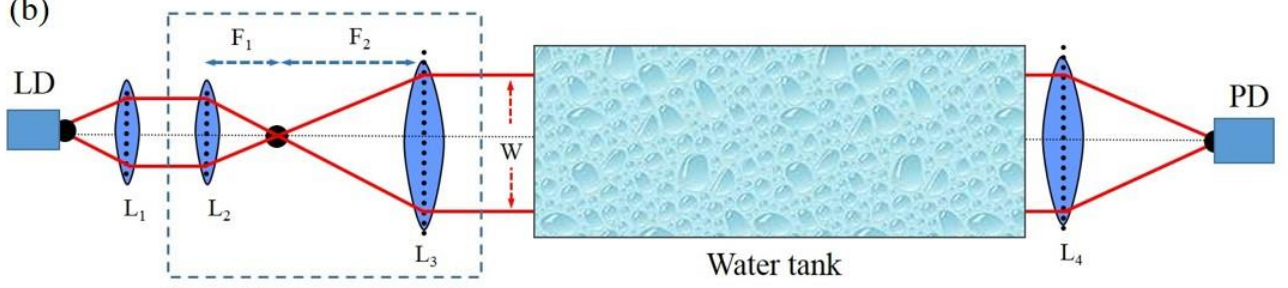

Keplerian beam expander

Fig. 1. Experimental setup for performance evaluation of UWOC in the presence of different air bubble sizes, (a) actual photograph: (i) LP520-SF15 LD, (ii) water tank, (iii) DET36A photodetector, (iv) S120VC power meter, (v) MDO3104 oscilloscope; (b) schematic: laser diode

(LD), lens $\left(\mathrm{L}_{1}=25.4, \mathrm{~L}_{2}=15.29, \mathrm{~L}_{3}=\right.$ variable, and $\left.\mathrm{L}_{4}=75 \mathrm{~mm}\right)$, focal length $\left(\mathrm{F}_{1}, \mathrm{~F}_{2}\right)$, beam size $(\mathrm{W})$, and photodetector $(\mathrm{PD})$.

A $15 \mathrm{~mW}$, single-mode continuous-wave (cw) $520 \mathrm{~nm}$ fiber-pigtailed green LD (LP520-SF15) is used as the transmitter. The L-I-V characteristics of the transmitter LD at room temperature are presented in Fig. 2(a). The LD exhibits a threshold current of around $58 \mathrm{~mA}$ and an optical power of $6.9 \mathrm{~mW}$ at $83 \mathrm{~mA}$ injection current. Fig. 2(b) presents the emission spectra of the LD under different injections currents measured using a high-resolution spectrometer (Ocean Optics HR4000). A peak emission wavelength of $515.3 \mathrm{~nm}$ was measured at $75 \mathrm{~mA}$. The laser beam was collimated by a $25 \mathrm{~mm}$ plano-convex lens (Thorlabs LA1951-A) and directed down the axis of the water tank. The collimated beam size was $4 \mathrm{~mm}$ in diameter. The average incident power on the water column was around $1.2 \mathrm{~mW}$, which ensures that a considerable amount of power reach the receiver unit.
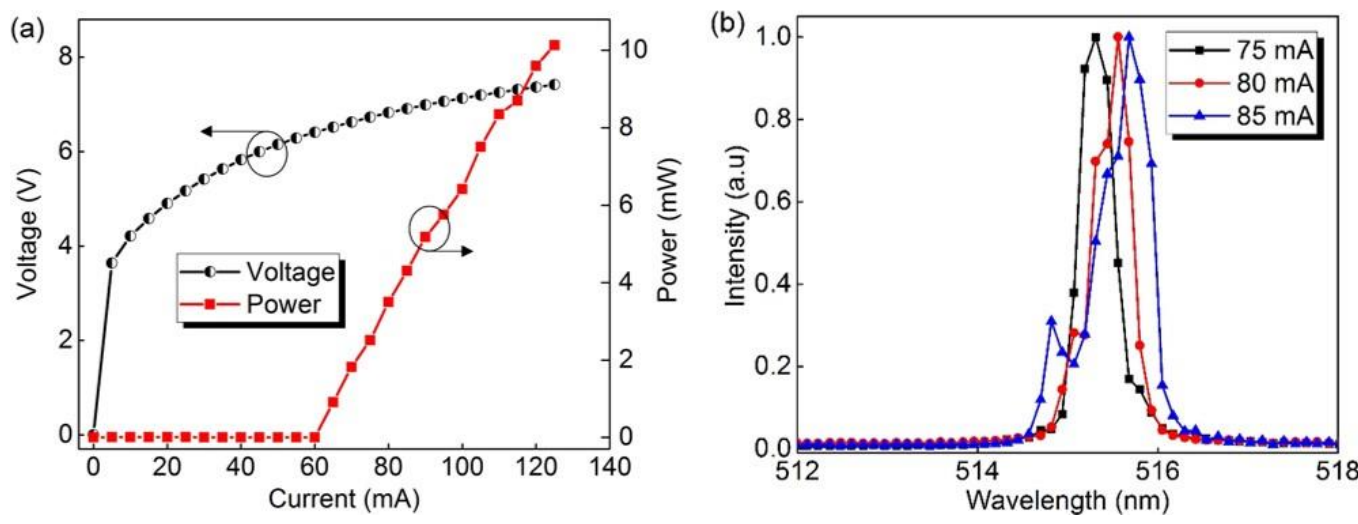

Fig. 2. L-I-V characteristics (a) and emission spectra (b) of the $520 \mathrm{~nm}$ green LD. 
Light passing through the exit optical window was collected using a $75 \mathrm{~mm}$ lens (Thorlabs LA1608) and focused into an $80 \mathrm{MHz}$ bandwidth silicon PIN photodiode (PD) receiver for intensity fluctuation measurements. The relevant specifications of the PD (Thorlabs DET36A) include an active diameter of $13 \mathrm{~mm}^{2}$, a responsivity of around 0.19 $\mathrm{A} / \mathrm{W}$ at $520 \mathrm{~nm}$ and a noise equivalent power (NEP) of $0.016 \mathrm{pW} / \mathrm{Hz}^{1 / 2}$. The output of the PD was fed into a Tektronix mixed domain oscilloscope (MDO3104) with a sampling rate of $5 \mathrm{GSa} / \mathrm{s}$ to monitor and measure the intensity of the received signals. For bit error rate (BER) measurements, we used an avalanche photodetector (APD) module as a receiver. The APD (Menlo Systems APD210) has an active diameter of $0.5 \mathrm{~mm}$, a responsivity of around $13 \mathrm{~A} / \mathrm{W}$ at $520 \mathrm{~nm}$ and a noise equivalent power (NEP) of $0.4 \mathrm{pW} / \mathrm{Hz}^{1 / 2}$. The BER performance of the UWOC system was analyzed using a high-performance bit error rate tester (Agilent J-BERT N4903B).

The water turbidity in the tank was varied by adding different amounts of Maalox antacid, whose ingredients are aluminum hydroxide and magnesium hydroxide. Maalox is known to provide excellent scattering similar to real ocean particles and commonly used in laboratory experiments to simulate different ocean water types [7, 24]. Maalox concentrations corresponding respectively to clear $\left(c=0.151 \mathrm{~m}^{-1}\right)$ and Harbor II $\left(c=2.19 \mathrm{~m}^{-1}\right)$ waters were calculated and added based on reference [7]. The values of absorption coefficient a, scattering coefficient $b$, and total attenuation coefficient $c=\mathrm{a}+\mathrm{b}$, for clear and harbor II waters are listed in Table 1 .

Table 1. Representative absorption, scattering and total attenuation coefficient values as measured by [24]

\begin{tabular}{lccc}
\hline Water Type & $\mathrm{a}\left(\mathrm{m}^{-1}\right)$ & $\mathrm{b}\left(\mathrm{m}^{-1}\right)$ & $\mathrm{c}\left(\mathrm{m}^{-1}\right)$ \\
\hline Clear water & 0.114 & 0.037 & 0.151 \\
Harbor II & 0.366 & 1.824 & 2.19 \\
\hline
\end{tabular}

\section{Results and Discussion}

We first investigated underwater wireless optical channel characteristics without air bubbles by evaluating the BER performance of the UWOC system under four different pump flow rates. Using two type-K thermocouple based temperature probes, the temperature variations within the water tank were constantly monitored and found to be very stable. Fig. 3(a) shows the BER performance as a function of received optical power at data rate of $0.622 \mathrm{Gbit} / \mathrm{s}$ for both clear ocean and Harbor II waters with the corresponding eye diagrams presented in Fig. 3(b) and Fig. 3(c), respectively. The eye diagrams are wide open indicating an error-free communication link. As we can clearly see from Fig. 3(a), the measured values of BER are almost equal for all four water pump flow rates in the case of clear ocean water as well as Harbor II water. We also analyzed the effect of high data rate on BER for all four water flow conditions as shown in Fig. 4. Similarly, the measured values of BER are almost equal for both clear ocean and turbid Harbor II waters.

From Fig. $3 \& 4$, we can infer that circulating and moving water has no effect on the performance of UWOC links as expected. This is due to the fact that although the circulation of our pump is sufficiently chaotic similar to the flows in natural bodies of water being in random with eddy-current like motions dominated by inertial-vortex forces [25], there are no changes in the index of refraction due to localized temperature or salinity variations within the water tank, resulting in a homogeneous optical channel. In such a homogeneous underwater channel, the optical properties are not affected and can not be labeled as turbulent channel when light beam is propagating through despite the water is in highly random motions, sufficiently chaotic and confused. 

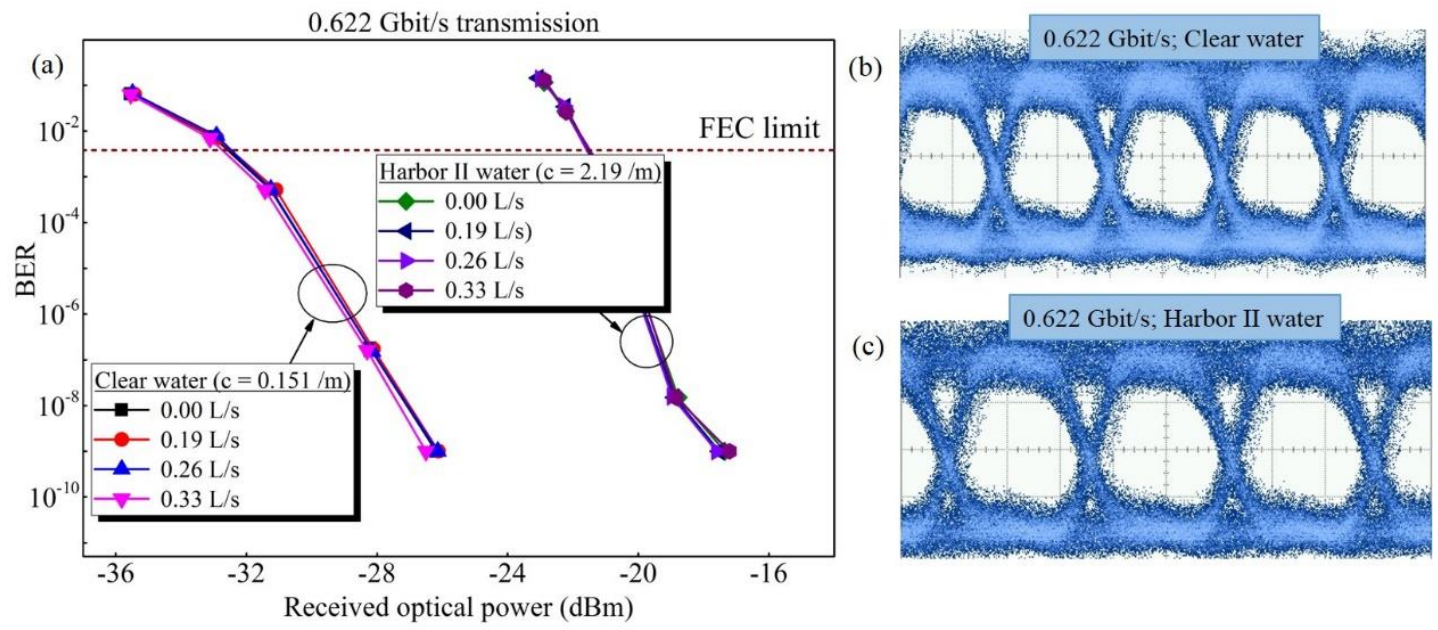

Fig .3. (a) Plot of BER as a function of received optical power at data rate of $0.622 \mathrm{Gbit} / \mathrm{s}$ for clear ocean and Harbor II waters, (b) (c) corresponding eye diagrams.

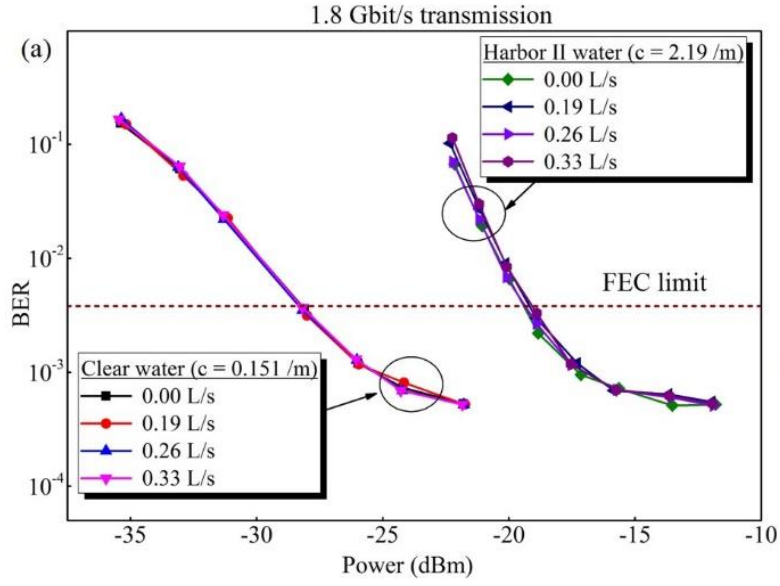

(b)

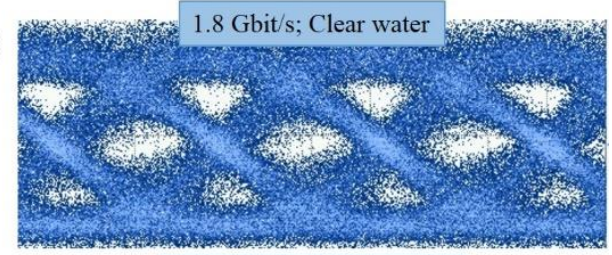

(c)

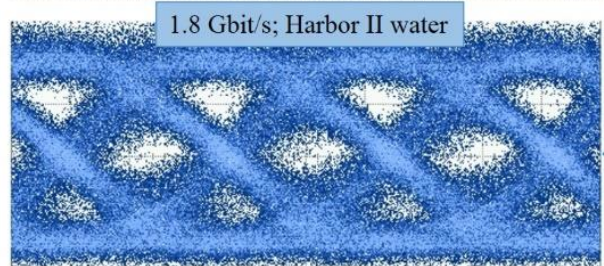

Fig. 4. (a) Plot of BER as a function of received optical power at data rate of $1.8 \mathrm{Gbit} / \mathrm{s}$ for clear ocean and Harbor II waters, (b) (c) corresponding eye diagrams.

To investigate the effect of different air bubble sizes on UWOC links, we introduced a small amount of air into the water tank as discussed in section 2. By placing the pump in front of the bubble generator, we created four different air bubble populations depending on the pump flow rate, namely extra-large (x-large), large, medium, and small bubbles corresponding to 0.00 (pump off), $0.19,0.26$, and $0.33 \mathrm{~L} / \mathrm{s}$, respectively. For each pump rate, bubbles photographic images were collected using Nikon D5500 camera fitted with Tamron AF $90 \mathrm{~mm} \mathrm{f} / 2.8$ macro lens that looked directly into the air pipe. Subsequently, the images were processed on ImageJ software to calculate both size spectra and the frequency of their occurrences. Fig. 5 shows photographs and corresponding histograms of the four air bubble populations. As the pump rate increases, the air bubble population becomes smaller in size and larger in number as shown on the inset images because the high pump rate helps break up the bubbles into smaller bubbles. For instance, when the pump is off $(0.00 \mathrm{~L} / \mathrm{s})$, there are approximately 22 air bubbles with an average area of $28 \mathrm{~mm}^{2}$ whereas in the case of $0.33 \mathrm{~L} / \mathrm{s}$, there are over 280 air bubbles with an estimated average area of $5.8 \mathrm{~mm}^{2}$. 

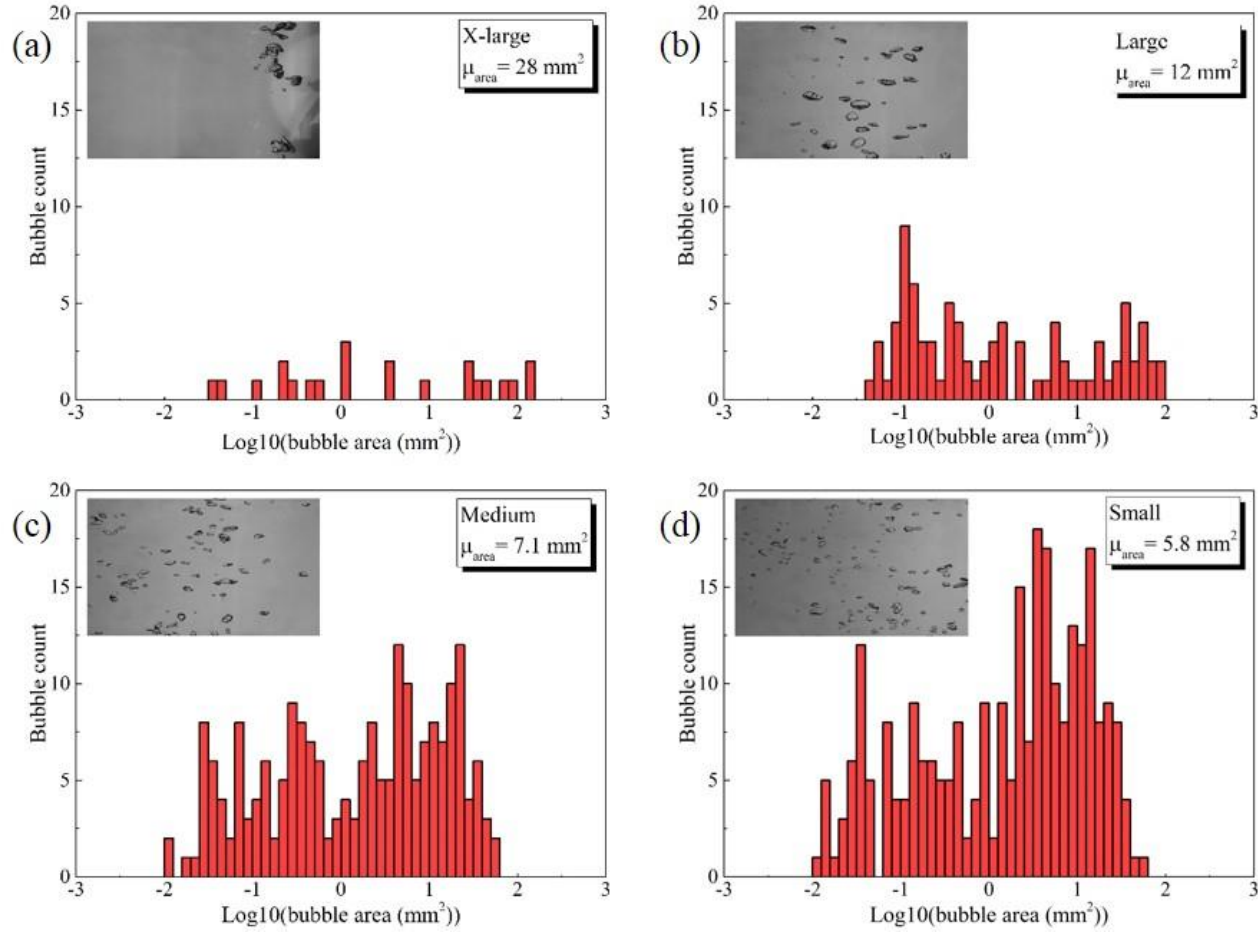

Fig. 5. (a) (d) Actual photographs of four different air bubbles in the water channel under four different water circulation levels with corresponding bubble size histograms.

Fig. 6(a) illustrates the received signal through the bubbly Harbor II water under different bubble sizes as captured by an oscilloscope with a sampling rate of $50 \mathrm{kS} / \mathrm{s}$. In the presence of air bubbles, the received signal severely fluctuates as evident from the figure. When the pump is off, the bubble size is large enough to completely obstruct the beam creating a deep fade which is a temporary failure of communication due to the severe drop in the channel signalto-noise ratio (SNR). It is worth noting that the frequency of obstruction (fluctuations) is less since the bubble population is less as depicted in Fig. 5(a). However, the variance ( $\left.I_{\max }-I_{\min }\right)$ of deep fade is large because at times the intensity drops down to zero. As the bubble breaking increases due to higher flow rates, the frequency of fluctuations increases gradually due to an increase in bubble population size. Moreover, the variance of fading actually decreases because as the bubbles are getting smaller, the beam becomes less susceptible to complete obstruction and more light reaches the receiver unit. Deep fade can be overcome by using an error-correcting code in conjunction with interleaving or simple diversity technique such as multiple input single output (MISO). In Fig. 6(b), the performance of the UWOC system is presented in terms of received versus transmitted optical power. We observe that the performance degrades significantly as the bubble size decreases. Again, this is the result of increase in the amount of air bubbles along the beam path that act as scatters. For instance, to achieve a received power of $-32 \mathrm{dBm}$, approximately a transmit power of $-12 \mathrm{dBm}$ is required in the case of extra-large bubbles. However, in the case of small bubbles, a minimum transmit power of about $-3 \mathrm{dBm}$ is required to achieve similar performance. These two figures (Fig. 6(a) \& Fig. 6(b)) show that the UWOC system design can be significantly changed depending on the density and size of air bubbles in the optical link. 

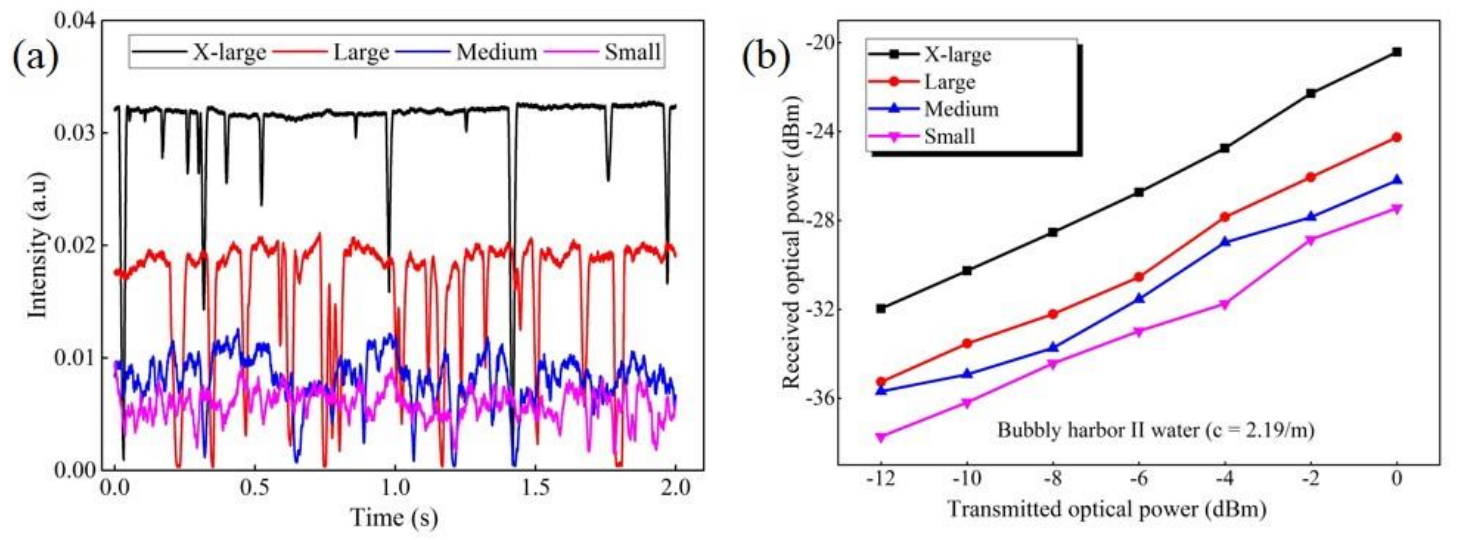

Fig. 6. Performance of UWOC link through bubbly Harbor II water under different bubble sizes: (a) intensity of received signal; (b) plot of received versus transmitted optical power.

To overcome the performance limitations posed by air bubbles, we propose and study the effect of beam expansion. A Keplerian beam expander was built from off-the-shelf components as depicted schematically in Fig. 1(b). The magnification $M$ of the beam expander is given by.

$$
M=\frac{F_{2}}{F_{1}}
$$

where $F_{1}$ and $F_{2}$ are the effective focal length of the entry and exit lenses, respectively. The originally collimated 4 $\mathrm{mm}$ beam was used as the input beam into the beam expander system. A mounted Rochester aspheric lens of 15.29 $\mathrm{mm}$ focal length (Thorlabs A260TM-A) was chosen as a fixed value of $F_{1}$. We utilized 25.4, 35, 50, and $60 \mathrm{~mm}$ focal length lens for $F_{2}$ to obtain an expanded beam of 6.6, 9.2, 13.1, and $15.7 \mathrm{~mm}$ beamwidth, respectively.

Received signal intensity measurements were performed at five different beam sizes under all four bubble sizes as shown in Fig. 7(a) - (d). Note that every plot is normalized to its own maximum. Two important observations can be made regarding Fig. 7. First, for a given bubble size, the variance of fading decreases as the beamwidth increases. A larger beam is less prone to deep fade as evident from Fig. 7(a) where the bubble size is the largest. As we can see from this figure, when the beamwidth reaches $13.1 \mathrm{~mm}$, the severity of drop becomes less (about $70 \%$ drop) compared to the other three smaller beam sizes where the intensity drops down to zero (up to $100 \%$ drop). Second, for a beamwidth of $9.2 \mathrm{~mm}$ or larger, the variance of fading smooths out as the bubble size decreases. For example, we observe nearly a flat curve in the case of $15.7 \mathrm{~mm}$ beam when the bubbles are small. As evident from Fig. 6(a) and Fig. 7, there is a tradeoff between mitigating the deep fade and the level of received intensity. Although the received intensity level decreased significantly, the low fading could enable a reliable communication in underwater wireless optical links. This figure also shows that system design depends on the relation between beamwidth and bubble distribution. The beamwidth should be carefully determined. Thus, beam expansion is a potential technique to improve the performance of UWOC links in the presence of air bubbles. 


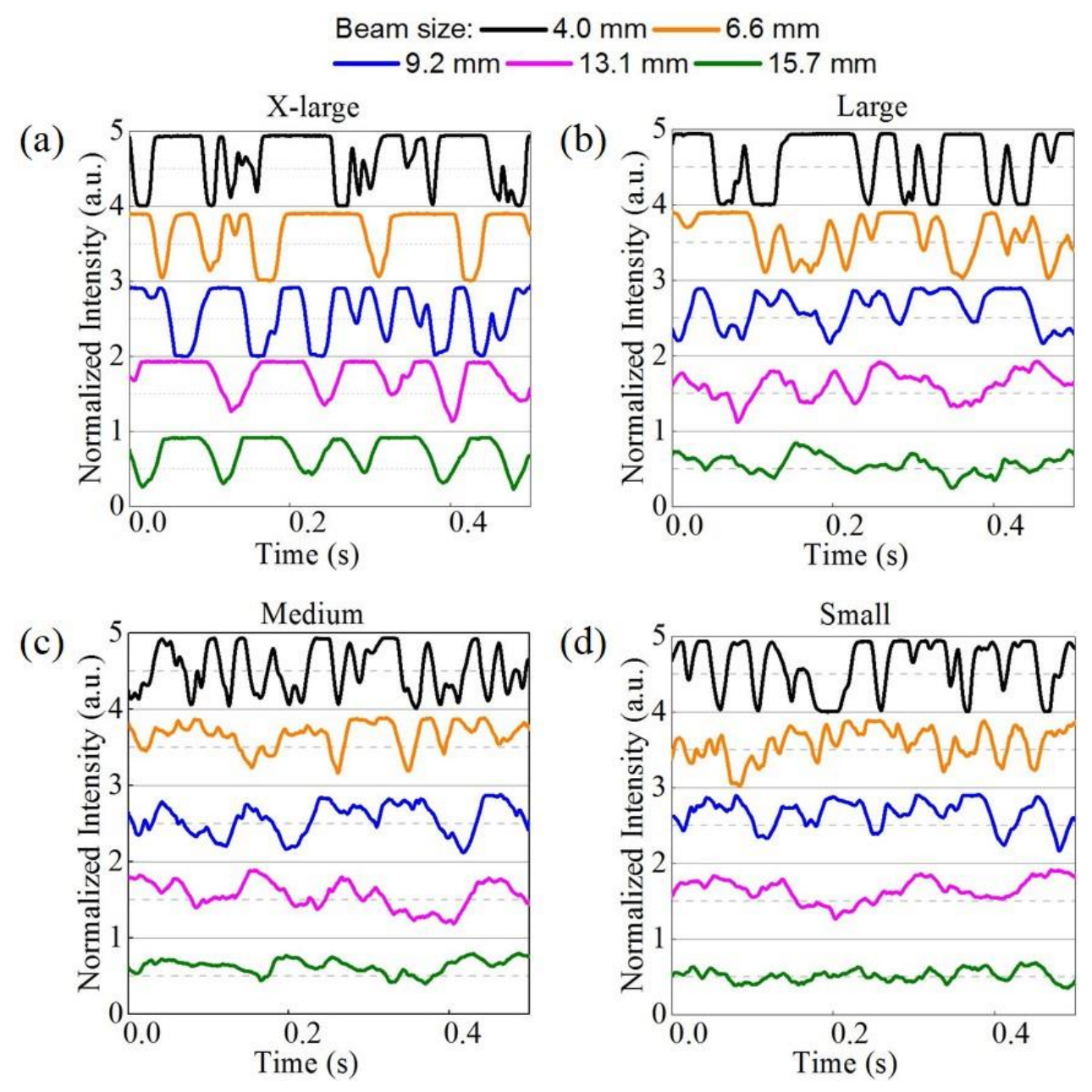

Fig. 7. (a) (d) Normalized intensity of received signal at different beam sizes and bubble sizes.

\section{Conclusion}

In this paper, we experimentally analyzed the performance of UWOC systems in the presence of different air bubbles sizes. Our measurement results reveal that air bubbles deteriorate the quality of the received signal leading to performance degradation of UWOC link. Large bubbles that are comparable to beamwidth in size can cause a total communication failure or deep fade. In the presence of small air bubbles, the optical beam is less susceptible to deep fade. We also showed that beam expansion is a potential technique to improve the performance degradation of UWOC links due to air bubbles. Our findings will assist engineers to design better transmitter systems for UWOC applications.

\section{Acknowledgment}

The authors gratefully acknowledge the financial support from Qatar National Research Fund (QNRF) grant through National Priority Research Program (NPRP) No. 8-648-2-273.

\section{References}

[1] D. Tsonev, H. Chun, S. Rajbhandari, J. J. D. McKendry, S. Videv, E. Gu, M. Haji, S. Watson, A. E. Kelly, G. Faulkner, M. D. Dawson, H. Haas, and D. O'Brien, "A 3-Gb/s single-LED OFDM-based wireless VLC link using a Gallium Nitride $\mu$ LED," IEEE Photonics Technol. Lett. 26(7), 637-640 (2014).

[2] Y.-C. Chi, D.-H. Hsieh, C.-T. Tsai, H.-Y. Chen, H.-C. Kuo, and G.-R. Lin, "450-nm GaN laser diode enables high-speed visible light communication with 9-Gbps QAM-OFDM,” Opt. Express 23(10), 13051-13059 (2015). 
[3] Chao Shen, Yujian Guo, Hassan M. Oubei, Tien Khee Ng, Guangyu Liu, Ki-Hong Park, Kang-Ting Ho, Mohamed-Slim Alouini, and Boon S. Ooi, "20-meter underwater wireless optical communication link with 1.5 Gbps data rate," Opt. Express 24, 25502-25509 (2016).

[4] H. H. Lu et al., "An 8 m/9.6 Gbps underwater Wireless optical communication system," in IEEE Photonics Journal, vol. 8, no. 5, pp. 1-7, Oct. 2016.

[5] Wei Lu, Liren Liu and Jianfeng Sun, "Influence of temperature and salinity fluctuations on propagation behaviour of partially coherent beams in oceanic turbulence," J. Opt. A: Pure Appl. Opt. 8, 1052-1058 (2006).

[6] B. M. Cochenour and L. J. Mullen, "Free-space optical communications underwater," in Advanced Optical Wireless Communication System, S. Arnon, J. Barry, G. Karagiannidis, R. Schober, and M. Uysal, eds. (Cambridge University Press, 2012), pp. 201-239.

[7] A. Laux, R. Billmers, L. Mullen, B. Concannon, J. Davis, J. Prentice, and V. Contarino, "The abc's of oceanographic lidar predictions: A significant step toward closing the loop between theory and experiment," J. Mod. Opt. 49, 439-451 (2002).

[8] W. Cox and J. Muth, "Simulating channel losses in an underwater optical communication system,” J. Opt. Soc. Am. A 31(5), 920-934 (2014).

[9] B. Cochenour, L. Mullen, and J. Muth, "Effect of scattering albedo on attenuation and polarization of light underwater," Opt. Lett. , vol. 35, no. 12 , pp. 2088-2090, June 2010

[10] H. M. Oubei, C. Li, K.-H. Park, T. K. Ng, M.-S. Alouini, and B. S. Ooi, "2.3 Gbit/s underwater wireless optical communications using directly modulated $520 \mathrm{~nm}$ laser diode," Opt. Exp., vol. 23, no. 16, pp. 20743-20748, 2015.

[11] K. Nakamura, I. Mizukoshi, and M. Hanawa, "Optical wireless transmission of 405 nm, 1.45 Gbit/s optical IM/DD-OFDM signals through a 4.8 m underwater channel,” Opt. Exp., vol. 23, no. 2, pp. 1558-1566, 2015.

[12] F. Hanson and S. Radic, "High bandwidth underwater optical communication," Appl. Opt. 47(2), 277-283 (2008).

[13] H. M. Oubei et al., "4.8 Gbit/s 16-QAM-OFDM transmission based on compact 450-nm laser for underwater wireless optical communication,” Opt. Exp., vol. 23, no. 18, pp. 23302-23309, 2015.

[14] Frank Hanson and Mark Lasher "Effects of underwater turbulence on laser beam propagation and coupling into single-mode optical fiber," Appl. Opt. 49(16), 3224-3230 (2010).

[15] D.J. Bogucki et al. "Comparison of near-forward light scattering on oceanic turbulence and particles,” Appl. Opt. 37(21), pp. 4669-4677 (1998).

[16] M. V. Jamali et al., "Statistical distribution of intensity fluctuations for underwater wireless optical channels in the presence of air bubbles," 2016 Iran Workshop on Communication and Information Theory (IWCIT), Tehran, Iran, 2016, pp. 1-6.

[17] R. M. Hagem, D. V. Thiel, S. G. O'Keefe, and T. Fickenscher, "The effect of air bubbles on an underwater optical communications system for wireless sensor network applications,” Microw. Opt. Technol. Lett., 54: 729-732, 2012.

[18] D. K. Woolf, Bubbles, "Encyclopedia of ocean sciences", Eds J.H. Steele, S.A. Thorpe and K.K. Turekian, Academic Press, pp. $352-357$ (2001).

[19] H. Medwin, “In situ acousticm easurementosf bubblep opulations in coastalo ceanw aters," J. Geophys Res., 75, 599-611, 1970.

[20] D. A. Kolovayev, "Investigation of the concentration and statistical size distribution of wind-produced bubbles in the near-surface ocean," Oceanology, Engl. Transl., 15, 659-661, 1976.

[21] B. D. Johnson, and R. C. Cooke, "Bubble populations and spectra in coastal waters: A photographic approach," J. Geophys. Res., 84, 3761$3766,1979$.

[22] D. M. Farmer, and D. D Lemon, “The influence of bubbles on ambient noise in the ocean at high wind speeds," J. Phys. Oceanogr. 14(11):1762-1778, 1984.

[23] D. C. Blanchard, and L. D. Syzdek, “Concentration of bacteria in jet drops from bursting bubbles,” J. Geophys. Res. 77, 5087-5099, 1972.

[24] T. J. Petzold, "Volume scattering functions for selected ocean waters," Scripps Institute of Oceanography Visibility Laboratory, Paper SIO Reference. 72-78 (1972).

[25] Carl H. Gibson, “Turbulence in the ocean, atmosphere, galaxy, and universe,” Appl. Mech. Rev 49(5), 299-315, 1996. 